\title{
ARTICLE
}

\section{The interface between neurology and psychiatry: the case of multiple sclerosis}

\author{
Eugene K. O. Wong, Rajeev Krishnadas \& Jonathan Cavanagh
}

Eugene Wong is an ST5 in general adult psychiatry at Parkhead Hospital, Glasgow. Rajeev Krishnadas is a clinical lecturer and honorary specialist registrar in general adult psychiatry at the Institute of Health and Wellbeing, University of Glasgow. Jonathan Cavanagh is a reader in psychiatry at the Institute of Health and Wellbeing, University of Glasgow. Correspondence Dr Eugene K. 0 . Wong, Anvil Centre, Parkhead Hospital, 81 Salamanca Street, Glasgow G31 5ES, UK. Email: eugene.wongs@gmail.com

\section{SUMMARY \\ There has been a surge in interest in the boundaries of psychiatry, both as a specialty in its own right and as a branch of medicine separate from neurology. Our article expands on this, giving examples of how recent developments in neuro- immunology can be beneficial for psychiatry, using multiple sclerosis (MS) as an example. We also provide a summary of literature on novel research in the treatment of depression using anti-inflammatory agents. Finally, we suggest approaches to the identification and management of major depressive disorder in patients with MS or other physical illnesses, and how this translates to general psychiatric practice. \\ DECLARATION OF INTEREST \\ J. C. has received grants from Biogen and Pfizer.}

Advancements in genetics and neuroimaging over the past few decades have allowed us to begin to understand the biological processes underlying many illnesses originally thought to be purely 'functional' (Kandel 1998). The pathophysiology behind diseases such as Parkinson's and epilepsy, for example - previously thought to be mental illnesses without any identifiable physical basis of diagnosis or treatment - are now more clearly understood. However, as happens to many of these 'functional' illnesses, the care and treatment of the majority of patients with Parkinson's disease and epilepsy have subsequently become the responsibility of neurologists.

More recently, the mass media has shown increasing interest in patients diagnosed with a psychiatric illness who have subsequently been found to have antibody-mediated encephalitis, and this has led to suggestions that a proportion of these patients may have an antibody-mediated aetiopathogenesis (Barry 2011; Lennox 2012). The optimism of our scientific abilities, together with the tendency for mental illness to be reclassified as neurological as biological understanding advances, has sparked concern for the survival of psychiatry as a specialty in itself (Oyebode 2011), and may well mark the beginning of an age in which the brain and the mind (even psychiatry and neurology) are each considered essential in the understanding of the other.

It is well known that there is an increased risk of developing major depressive disorder in a number of physical illnesses - for instance, it affects $30 \%$ of stroke survivors (Hackett 2005) and up to $17 \%$ of people with rheumatoid arthritis, with similar rates in cancer and cardiovascular disease (Dickens 2002). In multiple sclerosis (MS), the lifetime prevalence of major depressive disorder is as high as $50 \%$, with suicide rates of up to $15 \%$ (Lo Fermo 2010). In this article, we examine the pathophysiological process of depression, particularly in physical illness, using MS as an example.

The article is divided into three sections:

- a brief outline of MS and associated psychiatric symptoms;

- the relationship between major depressive disorder, immune abnormalities key to the aetiopathogenesis of MS, and psychosocial stress;

- approaches to the identification and management of major depressive disorder in MS and patients with comorbid physical illnesses, and how these translate to general psychiatric practice.

\section{Multiple sclerosis and associated psychiatric symptoms}

\section{Clinical presentation of MS}

Multiple sclerosis is a chronic inflammatory disease of the central nervous system (CNS) in which multifocal lesions, characterised by the demyelination and partial loss of axons, lead to debilitating neurological and psychiatric symptoms (Box 1). Although these inflammatory episodes usually resolve in the early stages of the disease, they culminate in progressive neurological deterioration over time. The 'typical MS patient' is a young adult with a few distinct episodes of focal neurological signs with partial resolution. There are three types of MS, based on clinical course: 
BOX 1 Neurological and neuropsychiatric symptoms of multiple sclerosis

Neurological symptoms (Luzzio 2011)

- Fatigue $(75 \%)$

- Paraesthesias

- Spinal cord symptoms - muscle cramping secondary to spasticity, bladder/bowel/sexual dysfunction

- Charcot's triad (dysarthria, ataxia, tremor)

- Pain (30-50\%)

- Optic neuritis (20-40\%)

- Trigeminal neuralgia

- Facial myokymia

- Partial acute transverse myelitis

- Diplopia (33\%)

- Heat intolerance

- Dysphasia

- Seizures

Neuropsychiatric symptoms (Ghaffar 2007)

- Depression (50\%)

- Bipolar disorder

- Euphoria (25\%)

- Pseudobulbar affect (10\%)

- Psychosis (2-3\%)

- Cognitive impairment (40-65\%)

- Relapsing-remitting (80\% of cases) - stable phases alternated with unpredictable relapses with no clear precipitant. Relapses can last from hours to months and tend to vary in severity. Remission can last for up to several years. New symptoms may occur in a relapse.

- Primary progressive (15\%) - a steadily worsening of symptoms and disability without any distinct pattern of relapses or remissions.

- Secondary progressive - around 20 years after initial diagnosis, relapsing-remitting MS may progress to this subtype, which is characterised by a steadily worsening condition.

In MS, adjustment and coping with intermittent and unpredictable disability is something that is present throughout the course of the disease.

\section{Epidemiology of MS}

The prevalence of MS is about 2.1 million worldwide, with women being around three times more likely to be affected than men. It also typically presents earlier in women than in men. A systematic review of incidence studies listed in Medline between 1996 and 2007 (Alonso 2008) estimated that the female-to-male ratio of MS incidence increased from 1.4 to 2.3 over that period. The reason for this is still unknown. Multiple sclerosis has also been proven to be more common in Western countries further away from the equator, with the highest reported rate being 300 per 100000 in the Orkney islands in Scotland (Simpson 2011). Although there are many hypothetical reasons for this finding - changes in ultraviolet radiation and vitamin D levels as one moves away from the equator, for example - researchers have yet to substantiate the link. Racial and ethnic differences also play an important role in susceptibility to this disease (Rosati 2001).

\section{Aetiopathogenesis of MS}

Although the cause of MS is still open to debate, current evidence points to an autoimmune aetiology, where the damage to the CNS results from molecular mimicry. The body's immune system becomes activated by an antigen such as a virus, but then cross-reacts with the proteins that are part of the body. Helper T cells, which normally activate and direct other cells in the immune system towards foreign cells, become activated to react towards self-cells instead. They secrete pro-inflammatory cytokines, which upregulate the production of adhesion molecules, thereby increasing immune cell adhesion to the endothelium and increasing the migration of these cells across the blood-brain barrier into the CNS. Myelin proteins within the CNS are then presented as the initial foreign antigen (e.g. a virus), triggering a cascade of events resulting in injury to myelin and oligodendrocytes (O'Connor 2001). The most commonly implicated pro-inflammatory cytokines are interferon-gamma (IFN- $\gamma$ ), tumour necrosis factor-alpha (TNF- $\alpha$ ), and interleukins IL-1 $\beta$, IL-6 and IL-12 (Mohr 2006). Keep this in mind as we will expand on it later in the text.

Treatment for MS is based on the premise of this immunopathogenesis and works mainly by modulating the immune system.

\section{Neuropsychiatric symptoms in MS}

Multiple sclerosis is associated with a host of neuropsychiatric symptoms, such as depression, euphoria, pseudobulbar affect, psychosis and cognitive impairment (Box 1). Depression is the most common symptom by far, with an estimated lifetime prevalence of $50-55 \%$ and a median point prevalence of $28 \%$, based on a literature review (Arnason 2009). Not only are these figures considerably higher than rates in the general population, they also suggest that depression occurs more frequently or perhaps lasts longer than in the general population. In 
fact, among those with MS, depressive symptoms are as common as some classic (neurological) symptoms of the disorder (Box 1). Despite this, depression is not conventionally considered to be a core symptom of MS and may often be underdiagnosed. To complicate things further, four of the nine symptoms of depression in DSM-IV are also common in people with MS who are not depressed - fatigue, psychomotor retardation, decreased concentration and sleep disturbance (Mohr 1997). It can therefore be difficult to tease out whether these symptoms are caused by depression or by MS itself.

Depressive symptoms have been observed to occur before neurological symptoms of MS. Byatt, et al (2011) reported a 15\% incidence of depression 6 to 12 months prior to the onset of neurological symptoms. Depression in MS has also been associated with structural and biochemical changes in the CNS (indicative of the beginning of a relapse) even before neurological changes occur, suggesting a possible role for depression as a prodrome to MS. These findings are supported by evidence that changes in the immune system occur before episodes of depression in people with diagnosed MS (Foley 1992). This sequence would suggest that inflammation may play a role in the aetiopathogenesis of depression in people with MS.

The Goldman Consensus Group (2005) - a panel of experts on depression and MS - reported that depression in MS significantly affects function in several domains: people with MS depression perform poorly compared with non-depressed MS controls on standardised measures of cognitive function and quality of life. They also experience disruption in social support and family systems beyond what can be attributed to the disease alone. A depressive mindset can influence a patient's decision to start immunomodulatory treatment and to continue with treatment once it has begun. Depression has been shown to be the main determinant of quality of life in MS (D'Alisa 2006).

As mentioned above, up to $55 \%$ of people with MS develop depression. So should depression be considered one of the core symptoms of MS i.e. is the separation of physical and psychiatric symptoms necessary?

\section{Major depressive disorder, psychosocial stress and the central role of the immune system in their relationship}

In recent years, there has been increasing evidence to suggest that depression in MS is mediated by the same pathological processes that cause the development of this disease. For example, a higher rate of depression and suicide has been shown to be associated with periods of exacerbation in MS (Kroencke 2001). The following sections explore this finding.

\section{The role of pro-inflammatory cytokines}

Although traditionally considered an 'immuneprivileged' organ, we now know that the brain is susceptible to immune-mediated insult. Cytokines (cyto - cell, kinos - movement) are immunomodulatory agents which are produced by all nucleated cells and used to mediate cellular communication within the immune system. They are formed and released as a 'cascade', where induction of one of the molecules can trigger the induction/ activation of a number of others. Cytokines are present in the brain. They are 'gliotransmitters' that act on a number of receptors, and are thought to be key in several brain functions. Where the primary focus of immune activity is the brain, as it is in MS, the cytokines may be produced in the brain itself. Cytokines produced outside of the CNS also have the potential to affect the brain via a number of mechanisms (Capuron 2011; Krishnadas 2012):

- crossing through 'leaky' regions in the bloodbrain barrier

- active transport

- transmission of signals along the afferent vagal pathway

- entry of activated monocytes from the periphery of this pathway

- acting on the endothelial lining of the bloodbrain barrier.

Current research points towards a relationship between pro-inflammatory cytokines and depressive symptomatology. Maes (1999) describes increased IL-1 $\beta$, IL- 6 , TNF- $\alpha$ and IFN- $\gamma$, increased acute-phase proteins, and lower plasma tryptophan in depressed patients. Similarly, inflammation in the CNS leads to the activation of brain microglia and subsequently elevated proinflammatory factors (Qin 2007).

Cytokine expression is abnormally elevated in MS, as it is an inflammatory condition. However, an even higher expression of TNF- $\alpha$ - a proinflammatory cytokine implicated in depression was found in the blood of patients with MS whose depression scores were higher (Kahl 2002). This correlates the severity of MS depression with the level of cytokine secretion in the disease.

Last, direct administration of cytokines has been shown to cause depressive symptoms (Table 1). These symptoms usually lag behind the appearance of physical symptoms of cytokine administration and tend to linger after physical symptoms have abated, thus making it less likely 
that they arise as a result of physical discomfort (Capuron 2002).

\section{Psychosocial stress}

Psychosocial stress is a recognised risk factor for depression, be it a precipitant or a perpetuator, especially in those with a genetic predisposition. The role of stress in the development of MS depression was first described by Charcot (who was also the first person to recognise MS as a separate disease, which he called sclerose en plaques, in the late 1800s). He related grief, vexation and adverse changes in social circumstance to the onset of MS (Charcot 1877). More recently, Mohr \& Pelletier (2006) noted a clinical, though not statistically significant, relationship between stress and exacerbation of MS.

This stress does not simply arise from having to cope with MS. It is also strongly associated with the underlying disease process, which separates MS from other neurodegenerative disorders. For example, to determine whether certain CNS diseases are related to specific behavioural abnormalities, Schiffer \& Babigian (1984) examined whether 368 hospital patients with MS, 402 patients with temporal lobe epilepsy (TLE) and 124 patients with amyotrophic lateral sclerosis (ALS) had had contact with psychiatric services. They found that the prevalence of psychiatric contact for patients with MS and TLE was similar (19.3\% v. 22.9\%), and significantly higher than the rate for ALS (4.8\%). Patients with MS also had a significantly higher rate of depression than the other two groups. With the development of depression as a direct effect of brain pathology in mind, it is interesting to observe that psychiatric contact, and thus psychiatric symptoms, was more prevalent in the two diseases that affect the CNS than in the disease of motor neurons.

In addition to this, as mentioned earlier, depression tends to manifest before the onset of physical symptoms, at episodes of immune activation. Byatt et al (2011) report that in up to $75 \%$ of patients with major depressive disorder later found to have MS, the MS diagnosis was delayed because depression had been diagnosed.

Möller et al (1994) found no correlation between the severity of MS and the diagnosis of depression, concluding that the level of disability caused by MS is a poor predictor of the development of depression. An evaluation of peer support groups for people with MS reported that they did not result in consistent improvement in either depression or quality of life. Indeed, the authors concluded that such groups may actually be detrimental to those with better mental health (Messmer Uccelli 2004)

\begin{tabular}{|c|c|c|}
\hline Immunotherapy & $\begin{array}{l}\text { Clinical condition } \\
\text { treated }\end{array}$ & $\begin{array}{l}\text { Neuropsychiatric } \\
\text { side-effects }\end{array}$ \\
\hline Interferon alpha (IFN- $\alpha$ ) & $\begin{array}{l}\text { Cancer } \\
\text { Multiple sclerosis } \\
\text { Chronic hepatitis C } \\
\text { Other viral infections }\end{array}$ & $\begin{array}{l}\text { Fatigue } \\
\text { Psychomotor slowing } \\
\text { Depressed mood } \\
\text { Anxiety } \\
\text { Social withdrawal } \\
\text { Irritability } \\
\text { Anorexia } \\
\text { Cognitive disturbances (mental } \\
\text { slowing, lack of concentration, } \\
\text { memory impairment) }\end{array}$ \\
\hline Interferon beta (IFN- $\beta$ ) & Multiple sclerosis & $\begin{array}{l}\text { Fatigue } \\
\text { Depressed mood } \\
\text { Cognitive impairment }\end{array}$ \\
\hline Interleukin 1 (IL-1) & (Metastatic) cancer & Cognitive impairment \\
\hline Interleukin 2 (IL-2) & (Metastatic) cancer & $\begin{array}{l}\text { Fatigue } \\
\text { Anhedonia } \\
\text { Dysphoria } \\
\text { Cognitive impairment } \\
\text { (mental slowing) }\end{array}$ \\
\hline $\begin{array}{l}\text { Tumour necrosis factor alpha } \\
\text { (TFN- } \alpha \text { ) }\end{array}$ & Cancer & $\begin{array}{l}\text { Fatigue } \\
\text { Anorexia }\end{array}$ \\
\hline
\end{tabular}

After Schiepers 2005, with permission.

- an unexpected finding if depression is seen as a reaction to having to cope with the disability inherent in MS. However, a pilot study published in the same year reported a significant decrease in IFN- $\gamma$ in people with MS depression who had better social support (Mohr 2004).

Although equivocal, research suggests that depression in MS is just as likely to be due to the disease process as it is to psychosocial factors.

\section{The interaction between the HPA axis, cytokines} and psychosocial stress in MS depression

In normal hypothalamic-pituitary-adrenal (HPA) axis activity, corticotrophin-releasing hormone (CRH) secreted from the hypothalamus stimulates the pituitary gland to secrete adrenocorticotrophic hormone (ACTH). This hormone then stimulates the adrenal glands to secrete glucocorticoids (cortisol), which interact with corticosteroid receptors found in almost every tissue in the body. The main function of cortisol is in the regulation of energy metabolism. Cortisol also binds to glucocorticoid receptors in the hippocampus, amygdala and frontal lobes to inhibit its own secretion by downregulating $\mathrm{CRH}$ release from the hypothalamus.

In both depression and MS, there is overactivity of the HPA axis, leading to chronic hypercortisolaemia (Young 1991). The negative feedback mechanism is disrupted in depression as glucocorticoid receptor numbers and function are downregulated, leading to $\mathrm{CRH}$ transcription 
a. The same can be said for depression rates across the general population. In the epidemiological studies cited at the beginning of our article, the lifetime prevalence of depression of up to $55 \%$ would have been measured using various criteria, from self-assessment to structured diagnostic interviews. and, ultimately, increased cortisol secretion (Webster 2002). The majority of depressed people therefore have persistently elevated urinary, blood and CSF cortisol (Holsboer 2000). Successful antidepressant treatment blunts the overactivity of the HPA axis and reverses hypercortisolaemia (Holsboer 2000).

In MS, cytokines (such as IL-1, IL-6 and TNF) secreted during immune system activation have been shown to downregulate hypothalamic and pituitary glucocorticoid receptors just as in depression (Turnbull 1999). This can occur directly - through induced corticosteroid receptor resistance in the hypothalamus and pituitary gland (Miller 1999) - or indirectly, through activation of the indolamine pathway, which diverts tryptophan away from the serotonin pathway towards the production of quinolinic acid, a potent $N$-methyl-Daspartate (NMDA) receptor agonist. This has also been described as a cause of hippocampal atrophy and loss of corticosteroid receptors (Wichers 2004). Higher baseline HPA reactivity has been associated with an increased risk of MS disease progression (Gold 2005).

A study by Mohr \& Cox (2001) showed reductions in T-cell production of IFN- $\gamma$ in people with MS who were recovering from depression. Although these results did not reach statistical significance, the relationship between the decrease in IFN- $\gamma$ and the improvement in depressive cognitions was significant.

\section{Management of major depressive disorder in MS}

\section{Goldman Consensus guidelines}

A statement by the Goldman Consensus Group (2005) suggests an integrated approach based on a combination of antidepressants and cognitivebehavioural therapy (CBT): 'Treatment plans for depression among MS patients should be individualized, using psychotherapeutic, psychopharmacologic, or integrated approaches, depending upon individual circumstances, and preferences.'

The statement describes studies that have reported both group and individual CBT to be efficacious in the treatment of depressive symptoms in MS (Larcombe 1984; Foley 1987). Insightoriented therapy has also been shown to help with depression in hospital in-patients with MS when compared with a control group (Crawford 1985). It is worth noting that these studies did not require an official diagnosis of depression as an inclusion criterion, but rather measured relative levels on depression scoring instruments such as the Beck Depression Inventory (BDI) and Hamilton Rating
Scale for Depression (HRSD). ${ }^{\text {a }}$ However, it can be concluded that these psychological therapies have been shown to improve depressive symptoms.

\section{Cochrane Database}

In the most recent systematic review of depression in MS on the Cochrane Database (Koch 2011), only two trials totalling 70 participants were robust enough to be included. Although these trials suggested efficacy of paroxetine and desipramine in the treatment of MS depression, the difference from placebo was not statistically significant.

The Cochrane Collaboration also reviewed the efficacy of psychotherapy for treating people with MS depression (Thomas 2006). Evidence from six different studies suggests that CBT is useful in helping individuals with depression and equipping them with ways of coping with having MS. However, there were no studies assessing the differences between group and individual therapy or when it would be most appropriate to deliver these treatments.

\section{NICE guidelines on treatment of depression in physical illness}

On a more general note, the National Institute for Health and Care Excellence (NICE) makes recommendations on the management of depression in adults with chronic physical health problems (National Collaborating Centre for Mental Health 2009). Owing to the higher likelihood of drug interactions and higher prevalence of subthreshold depressive symptoms in this group, they recommend a higher threshold for the prescription of antidepressant medication and favour psychosocial treatments such as peer support and various types of CBT, especially for those with mild to moderate depression. They do not offer specific recommendations for people with severe depression and comorbid chronic physical health problems, instead directing readers to the NICE guideline for depression (National Institute for Health and Clinical Excellence 2009).

\section{Novel research - the role of anti-inflammatory agents}

Given the numerous links that have been shown to exist between inflammation and depression, it would be reasonable to surmise that treatments with anti-inflammatory agents may be beneficial in depression. Although there is insufficient evidence for inflammation as the main cause of all depression, there is enough to entertain the possibility of inflammation being the primary aetiology for a possible subtype of depression. The papers discussed below examine the use 
of anti-inflammatory agents in the treatment of depression.

A prospective double-blind randomised placebocontrolled add-on study reported a significant improvement in depressive symptoms on the HRSD when celecoxib was added to reboxetine, compared with placebo $(n=20$ v. 20) (Müller 2006). Similarly, aspirin accelerated the onset of action of fluoxetine from 3 weeks to only 1 week in rats assessed using an escape deficit model of depression. Pre-stressed rats were significantly better at avoiding noxious stimuli only 1 week into the administration of fluoxetine with aspirin, compared with those who were given either fluoxetine or aspirin alone (Brunello 2006).

More recently, a retrospective cohort study compared the exposure of 345 Australian women to aspirin or statins against a control population (Pasco 2010). The authors reported a rate of major depressive disorder of 1.7 in 1000 person-years in exposed participants and 12.2 per 1000 personyears in those not exposed to aspirin or statins over the previous 10 years, suggesting a possible role for anti-inflammatory drugs in protecting against major depressive disorder in women. This is in line with previous research on statins, where long-term statin use has been associated with a reduced incidence of depression and anxiety (Young-Xu 2003) and a lower risk of developing depression after 1 year (Yang 2003).

Our own research has shown that treatment with the TNF inhibitor adalimumab led to a decrease in serotonin transporter binding by up to $20 \%$ (Cavanagh 2010). However, it is not clear how peripheral blockade of inflammation using large molecules such as etanercept or adalimumab (which do not cross the blood-brain barrier) effect change in central mechanisms (Krishnadas 2010).

Conversely, selective serotonin reuptake inhibitors (SSRIs) have been shown to induce and sustain remission of inflammation in patients with rheumatoid arthritis (Krishnadas 2011).

\section{Implications for training and clinical practice}

This article describes the relationship between neurology and psychiatry through MS and its neuropsychiatric manifestations. This is an area that is ever-expanding with the advancement of understanding into the pathophysiology of psychiatric illness. Physical illnesses with significant neuropsychiatric manifestations, such as MS, are convenient starting points for this. In the case of MS, there is growing evidence that inflammation is a significant player in the aetiology of depression, and this has led to research into novel ways of treating depression through the targeting of inflammatory pathways.

In terms of clinical psychiatry, current research suggests a potential role for anti-inflammatory agents as adjuncts to conventional antidepressants. Although it may be too early to adopt this as routine practice, it is worth being prepared for this change in treatment paradigm. More imminently, there could be an argument for lowering the threshold for prescribing antidepressants in patients with comorbid inflammatory conditions. It would also be worth keeping MS in mind when presented with a patient with depression coupled with unexplained neurological signs.

In terms of psychiatric training, there is possibly an argument for greater cross-specialty training as the line dividing psychiatry and neurology begins to blur. Looking back, we find that currently accepted neurological disorders such as Parkinson's disease, epilepsy, chorea, torticollis and narcolepsy were described as 'functional' a century ago (Gowers 1893). These disorders are now well within the realm of neurology. That said, even if we eventually succeed in understanding the biological processes behind all mental illness, we would still be left with having to define the 'abnormal behaviour' from which mental illness is inferred. This complex concept is dependent on many variables, including biological and psychological states, social norms, individual resilience, interpersonal support and current political climate (Wong 2013), making it very unlikely that all mental illness will be absorbed into neurology. However, we want to emphasise the importance of not neglecting the brain when treating the mind.

\section{References}

Alonso A, Hernan MA (2008) Temporal trends in the incidence of multiple sclerosis: a systematic review. Neurology 71: 129-35.

Arnason BG (2009) Multiple sclerosis and depression: a neuroimmunological perspective. In The Brain and Host Defense (Neurolmmune Biology, vol 9) (ed BG Arnason): 269-307. Elsevier.

Barry H, Hardiman 0, Healy DG, et al (2011) Anti-NMDA receptor encephalitis: an important differential diagnosis in psychosis. British Journal of Psychiatry 199: 508-9.

Brunello N, Alboni S, Capone G, et al (2006) Acetylsalicylic acid accelerates the antidepressant effect of fluoxetine in the chronic escape deficit model of depression. International Clinical Psychopharmacology 21: 219-25.

Byatt N, Rothschild AJ, Riskind P, et al (2011) Relationships between multiple sclerosis and depression. Journal of Neuropsychiatry \& Clinical Neurosciences 23: 198-200.

Capuron L, Gumnick JF, Musselman DL, et al (2002) Neurobehavioral effects of interferon-alpha in cancer patients: phenomenology and paroxetine responsiveness of symptom dimensions. Neuropsychopharmacology 26: 643-52.

Capuron L, Miller AH (2011) Immune system to brain signaling: neuropsychopharmacological implications. Pharmacology \& Therapeutics 130: $226-38$. 


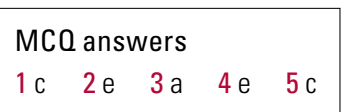

Cavanagh J, Paterson C, McLean J, et al (2010) Tumour necrosis factor blockade mediates altered serotonin transporter availability in rheumatoid arthritis: a clinical, proof-of-concept study. Annals of the Rheumatic Diseases 69: 1251-2.

Charcot J (1877) Lectures on Diseases of the Nervous System. New Sydenham Society.

Crawford JD, Mclvor GP (1985) Group psychotherapy: benefits in multiple sclerosis. Archives of Physical Medicine and Rehabilitation 66: 810-3.

D'Alisa S, Miscio G, Baudo S, et al (2006) Depression is the main determinant of quality of life in multiple sclerosis: a classificationregression (CART) study. Disability and Rehabilitation 28: 307-14.

Dickens C, McGowan L, Clark-Carter D, et al (2002) Depression in rheumatoid arthritis: a systematic review of the literature with metaanalysis. Psychosomatic Medicine 64: 52-60

Foley FW, Bedell JR, LaRocca NG, et al (1987) Efficacy of stressinoculation training in coping with multiple sclerosis. Journal of Consulting and Clinical Psychology 55: 919-22.

Foley FW, Traugott U, LaRocca NG, et al (1992) A prospective study of depression and immune dysregulation in multiple sclerosis. Archives of Neurology 49: 238-44.

Ghaffar 0, Feinstein A (2007) The neuropsychiatry of multiple sclerosis: a review of recent developments. Current Opinion in Psychiatry 20: 278-85.

Gold SM, Raji A, Huitinga I, et al (2005) Hypothalamo-pituitary-adrenal axis activity predicts disease progression in multiple sclerosis. Journal of Neuroimmunology 165: 186-91.

Goldman Consensus Group (2005) The Goldman Consensus statement on depression in multiple sclerosis. Multiple Sclerosis 11: 328-37.

Gowers WR (1893) A Manual of Diseases of the Nervous System (Reprinted 1970). Hafner.

Hackett ML, Anderson CS (2005) Predictors of depression after stroke: a systematic review of observational studies. Stroke 36: 2296-301.

Holsboer $F(2000)$ The corticosteroid receptor hypothesis of depression. Neuropsychopharmacology 23: 477-501.

Kahl KG, Kruse N, Faller $H$, et al (2002) Expression of tumor necrosis factor-alpha and interferon-gamma mRNA in blood cells correlates with depression scores during an acute attack in patients with multiple sclerosis. Psychoneuroendocrinology 27: 671-81.

Kandel ER (1998) A new intellectual framework for psychiatry. American Journal of Psychiatry 155: 457-69.

Koch MW, Glazenborg A, Uyttenboogaart M, et al (2011) Pharmacologic treatment of depression in multiple sclerosis. Cochrane Database of Systematic Reviews 2: CD007295

Krishnadas $R$ (2010) Etanercept for sleep in patients with alcohol use disorder - mechanisms need to be elucidated. Biological Psychiatry 67: e1.

Krishnadas R, Cavanagh J (2011) Sustained remission of rheumatoid arthritis with a specific serotonin reuptake inhibitor antidepressant: a case report and review of the literature. Journal of Medical Case Reports 5: 112.

Krishnadas R, Cavanagh J (2012) Depression: an inflammatory illness? Journal of Neurology, Neurosurgery \& Psychiatry 83: 495-502.

Kroencke DC, Denney DR, Lynch SG (2001) Depression during exacerbations in multiple sclerosis: the importance of uncertainty. Multiple Sclerosis 7: 237-42.

Larcombe NA, Wilson PH (1984) An evaluation of cognitive-behaviour therapy for depression in patients with multiple sclerosis. British Journal of Psychiatry 145: 366-71.

Lennox BR, Coles AJ, Vincent A (2012) Antibody-mediated encephalitis: a treatable cause of schizophrenia. British Journal of Psychiatry 200: 92-4.

Lo Fermo S, Barone R, Patti F, et al (2010) Outcome of psychiatric symptoms presenting at onset of multiple sclerosis: a retrospective study. Multiple Sclerosis 16: 742-8.

Luzzio C (2011) Multiple sclerosis clinical presentation. Medscape Reference (http://emedicine.medscape.com/article/1146199-clinical). Accessed 11 May 2013
Maes M (1999) Major depression and activation of the inflammatory response system. Advances in Experimental Medicine and Biology 461: 25-46.

Messmer Uccelli M, Mancuso Mohr L, Battaglia MA, et al (2004) Peer support groups in multiple sclerosis: current effectiveness and future directions. Multiple Sclerosis 10: 80-4.

Miller AH, Pariante CM, Pearce BD (1999) Effects of cytokines on glucocorticoid receptor expression and function: glucocorticoid resistance and relevance to depression. Advances in Experimental Medicine and Biology 461: 107-16.

Mohr DC, Goodkin DE, Gatto N, et al (1997) Depression, coping and level of neurological impairment in multiple sclerosis. Multiple Sclerosis 3 : 254-8.

Mohr DC, Cox D (2001) Multiple sclerosis: empirical literature for the clinical health psychologist. Journal of Clinical Psychology 57: 479-99.

Mohr DC, Genain C (2004) Social support as a buffer in the relationship between treatment for depression and T-cell production of interferon gamma in patients with multiple sclerosis. Journal of Psychosomatic Research 57: 155-8.

Mohr DC, Pelletier D (2006) A temporal framework for understanding the effects of stressful life events on inflammation in patients with multiple sclerosis. Brain, Behavior, and Immunity 20: 27-36.

Möller A, Wiedemann G, Rohde U, et al (1994) Correlates of cognitive impairment and depressive mood disorder in multiple sclerosis. Acta Psychiatrica Scandinavica 89: 117-21.

Müller N, Schwarz MJ, Dehning S, et al (2006) The cyclooxygenase-2 inhibitor celecoxib has therapeutic effects in major depression: results of a double-blind, randomized, placebo controlled, add-on pilot study to reboxetine. Molecular Psychiatry 11: 680-4.

National Collaborating Centre for Mental Health (2009) Depression in Adults with a Chronic Physical Health Problem: Treatment and Management (NICE Clinical Guideline 91). National Institute for Health and Clinical Excellence.

National Institute for Health and Clinical Excellence (2009) Depression in Adults: The treatment and Management of Depression in Adults (Update) (NICE Clinical Guideline 90). NICE.

O'Connor KC, Bar-Or A, Hafler DA (2001) The neuroimmunology of multiple sclerosis: possible roles of $\mathrm{T}$ and $\mathrm{B}$ lymphocytes in immunopathogenesis. Journal of Clinical Immunology 21: 81-92.

Oyebode F, Humphreys M (2011) The future of psychiatry. British Journal of Psychiatry 199: 439-40.

Pasco JA, Jacka FN, Williams LJ, et al (2010) Clinical implications of the cytokine hypothesis of depression: the association between use of statins and aspirin and the risk of major depression. Psychotherapy and Psychosomatics 79: 323-5.

Oin L, Wu X, Block ML, Liu Y, et al (2007) Systemic LPS causes chronic neuroinflammation and progressive neurodegeneration. Glia 55 : 453-62.

Rosati $G$ (2001) The prevalence of multiple sclerosis in the world: an update. Neurological Sciences 22: 117-39.

Schiepers OJ, Wichers MC, Maes M (2005) Cytokines and major depression. Progress in Neuro-Psychopharmacology and Biological Psychiatry 29: 201-17.

Schiffer RB, Babigian HM (1984) Behavioral disorders in multiple sclerosis, temporal lobe epilepsy, and amyotrophic lateral sclerosis. An epidemiologic study. Archives of Neurology 41: 1067-9.

Simpson S Jr, Blizzard L, Otahal P, et al (2011) Latitude is significantly associated with the prevalence of multiple sclerosis: a meta-analysis. Journal of Neurology, Neurosurgery \& Psychiatry 82: 1132-41.

Thomas PW, Thomas S, Hillier C, et al (2006) Psychological interventions for multiple sclerosis. Cochrane Database of Systematic Reviews 1: CD004431

Turnbull AV, Rivier CL (1999) Regulation of the hypothalamic-pituitaryadrenal axis by cytokines: actions and mechanisms of action. Physiological Reviews 79: 1-71. 
Webster MJ, Knable MB, O'Grady J, et al (2002) Regional specificity of brain glucocorticoid receptor mRNA alterations in subjects with schizophrenia and mood disorders. Molecular Psychiatry 7: 985-94.

Wichers MC, Maes M (2004) The role of indoleamine 2,3-dioxygenase (IDO) in the pathophysiology of interferon-alpha-induced depression. Journal of Psychiatry and Neuroscience 29: 11-7.

Wong EKO (2013) Borderline personality disorder in the east. Asian Journal of Psychiatry 6: 80-1.
Yang CC, Jick SS, Jick H (2003) Lipid-lowering drugs and the risk of depression and suicidal behavior. Archives of Internal Medicine 163 : 1926-32.

Young EA, Haskett RF, Murphy-Weinberg V, et al (1991) Loss of glucocorticoid fast feedback in depression. Archives of General Psychiatry 48 : 693-9.

Young-Xu Y, Chan KA, Liao JK, et al (2003) Long-term statin use and psychological well-being. Journal of the American College of Cardiology 42: $690-7$.

\section{MCQs}

Select the single best option for each question stem

\section{Regarding the interface between} psychiatry and general medicine:

a the prevalence of depression in people with inflammatory disorders is generally lower than in those without

b the main reason for the increase in major depressive disorder in patients with MS and rheumatoid arthritis is the burden of having to adapt to the associated disability

c Parkinson's disease, epilepsy and narcolepsy were considered 'functional' illnesses in the 1800 s

d current research suggests that all schizophrenia is antibody-mediated

e the resolution of psychiatric symptoms never leads to an improvement in comorbid physical illness.

\section{Which of the following is false - MS is} characterised by:

a chronic inflammation of the CNS, with demyelination and partial axonal loss

b a lifetime prevalence of depression of $50-55 \%$

c a higher rate of depression compared with amyotrophic lateral sclerosis

$\mathrm{d}$ higher levels of TNF- $\alpha$ e an overdiagnosis of depression due to overlapping symptoms such as fatigue, psychomotor retardation, poor concentration and sleep disturbance.

\section{Cytokines:}

a are elevated in major depressive disorder and inflammatory disorders such as MS

b do not cause symptoms of depression

c cannot cross the blood-brain barrier

$\mathrm{d}$ are contraindicated in the treatment of inflammatory conditions such as MS and hepatitis

e are not present in abnormally elevated levels in inflammatory conditions such as MS.

\section{Regarding psychosocial stress and MS}

a psychosocial stress is the result of having to cope with having MS rather than the underlying process of MS itself

b there is a strong correlation between the severity of MS and the diagnosis of depression

c there is an overactivity of the HPA axis in depression but not in MS

$\mathrm{d}$ the provision of social support to people with MS and depression usually leads to a significant improvement in mood

e patients with depression have been shown to have increased expression of TNF- $\alpha$.
5 Regarding novel treatments in depression:

a SSRIs have not been shown to have any effect on inflammation in those with rheumatoid arthritis

b aspirin has been shown to accelerate the onset of fluoxetine from 3 weeks to only 1 week in humans

c anti-inflammatory drugs such as aspirin or statins may have a role in protecting against major depressive disorder in women

d prophylactic statin use has been shown to reduce the lifelong incidence of schizophrenia

e anti-inflammatory drugs such as aspirin or statins may have a role in protecting against major depressive disorder in men. 